\title{
Ethisch-ökologische Finanzprodukte - eine Frage des Risikos?
}

\author{
Kapitalanleger können über den Kauf ethisch- \\ ökologischer Finanzprodukte zur Neubestim- \\ mung von Unternehmenszielen beitragen. \\ Verhaltenswissenschaftliche Erkenntnisse \\ zeigen, dass durch Änderungen in der \\ Beratung von Anlegern der Marktanteil \\ solcher Produkte erhöht werden könnte. \\ Von Uwe Demele und Gabriel Weber
}

\begin{abstract}
n den letzen zehn Jahren entfaltete sich eine äußerst rasante Entwicklung im ethisch-ökologischen Anlagesektor. Im gleichen Zeitraum belegten diverse empirische Studien, dass die Berücksichtigung von zukunftsverantwortlichen Kriterien bei der Geldanlage nicht zu Renditeeinbußen führt. Teilweise wurden gegenüber konventionellen Alternativen sogar positivere Effekte auf die finanzielle Wertentwicklung nachgewiesen (übersichtliche Darstellung bei Schäfer/ Stederoth 2002; Günther et al. 2004). Investmentfonds als dominante Ausprägung ethischökologischer Finanzprodukte erreichten im Jahr 2005 ein Marktvolumen von 1,2 Milliarden Euro, was einer dreißigprozentigen Steigerung gegenüber dem Vorjahr entspricht (Avanzi SRI Research/ SiRi Group 2005).
\end{abstract}

Die positiven Wachstumstendenzen dürfen jedoch nicht darüber hinweg täuschen, dass es sich bei prinzipiengeleiteten Anlageformen in Europa um einen Nischenmarkt handelt, der sich bislang in Größenordnungen von unter einem Prozent des Gesamtmarktes bewegt. Näher zu untersuchen ist daher, was Anleger(innen) neben rein finanzökonomischen Gründen daran hindert, ethisch-ökologisch zu investieren. So zeigt die Praxis besonders deutlich, dass die persönliche Anlageentscheidung zum Beispiel von affektiven und situativen Faktoren beeinflusst wird. Die zukünftige Marktentwicklung ethisch-ökologischer Finanzprodukte hängt entscheidend von der Beachtung und geschickten Handhabung dieser Einflussgrößen ab. Hierbei haben Beratungsgespräche in Banken, welche in diesem Beitrag näher betrachtet werden, einen zentralen Stellenwert.

\section{Ökologische Verhaltenslücke bei der Kapitalanlage}

In der gängigen Kapitalmarkttheorie wird von strikt rational handelnden Individuen ausgegangen, die in einer Welt ohne Transaktionskosten und Informationsasymmetrien ihren Nut- zen maximieren. Modellierungen unter diesen Annahmen legen das Menschenbild des homo oeconomicus zugrunde, welches sich als realitätsfern herausstellt (1). Emotional geprägt und von Transaktionskostenabwägungen beherrscht sind bereits die Vorüberlegungen vieler privater Bankkunden in Bezug auf die Wahl des Finanzinstituts. Sie verlassen sich dabei häufig auf ihr „Bauchgefühl“ und greifen bevorzugt auf die gewohnte und bequem erreichbare Hausbank zurück, ohne einen umfangreichen Anbietervergleich durchzuführen. Schon allein deswegen werden Grüne Finanzdienstleister, die gegenüber Finanzinstituten mit hoher Marktkapitalisierung derzeit noch eine geringe Kundenbindung aufweisen, vergleichsweise selten frequentiert (2).

Zwar befinden sich mittlerweile auch spezielle Aktienfonds mit ethisch-ökologischer Ausrichtung im Produktportfolio von Großbanken und konventionellen Finanzintermediären, allerdings werden sie zumeist nur als exotische Anlageform oder auf ausdrückliche Kundenanfrage angeboten. Weniger als ein Prozent der Bevölkerung hat bisher einen ethisch-ökologischen Fonds gekauft (Schoenheit 2005), obgleich nach der jüngsten, vom BMU herausgegebenen Studie zum Umweltbewusstsein in Deutschland 92 Prozent der Bevölkerung den Schutz der Umwelt als wichtig erachten (BMU 2004). In diesem Zusammenhang ist das Phänomen der ökologischen Verhaltenslücke, also das Auseinanderklaffen von geäußerter Umwelteinstellung und dem tatsächlichen Umweltverhalten, zu berücksichtigen. Umweltsoziologen beschäftigen sich mit dieser Diskrepanz und haben Erklärungsansätze entwickelt, welche Erkenntnisse der Neuen Institutionenökonomie und Transaktionskostentheorie einbeziehen.

Es wird angenommen, dass die Verhaltenswirksamkeit des Umweltbewusstseins am stärksten ausgeprägt ist, wenn die Kosten umweltorientierten Verhaltens niedrig sind (Diekmann/ Preisendörfer 2001). Der Kostenbegriff ist hierbei sehr weit gefasst, eingeschlossen sind auch nicht monetär messbare Unbequemlichkeiten im Rahmen von Transaktionen. Von einer LowCost-Situation für den Investor kann bei guter Erreichbarkeit ethisch-ökologischer Finanzintermediäre, geringem Informationsaufwand und unkomplizierten Abwicklungsmodalitäten ausgegangen werden (siehe Abbildung 1). Insgesamt wird deutlich, dass Umweltbewusstsein eine notwendige, aber noch keine hinreichende Voraussetzung für die umweltadäquate Anlageentscheidung ist.

Umweltpsychologen konzentrieren sich zur Erklärung von Einstellungs-Verhaltens-Zusammenhängen vornehmlich auf Wahrnehmungs- und Reflexionsprozesse umweltethischer $\rightarrow$ 
Abbildung 1: Low-Cost-These zum umweltorientierten Anlageverhalten

Low-Cost-Situation

Quelle: angelehnt an Diekmann/ Preisendörfer (2001:118)

Missstände. Je intensiver diese sind, desto gewisser ist umweltfreundliches Verhalten (Brucks 2004). Folgt man dieser Basishypothese, so ergeben sich, bezogen auf den Untersuchungsgegenstand, Voraussetzungen, die in der Summe ethischökologische Finanzengagements begünstigen. Kapitalanleger(innen) müssen sich zunächst der Tragweite ihrer persönlichen Anlageentscheidung bewusst werden. Dazu gehört das Wissen über die Weiterverwertung des Geldes im Kapitalmarkt und die Übernahme von Verantwortung für die beabsichtigte Investitionsstrategie. Wesentlich ist auch die Bereitschaft zur Mitgestaltung der Gesellschaft im Sinne einer intra- und intergenerationellen Gerechtigkeit. Es erscheint zudem plausibel, dass Kapitalanleger(innen) ethisch-ökologischen Finanzprodukten viel aufgeschlossener gegenüber stehen, wenn sie oder Menschen in ihrem persönlichen Umfeld von unternehmerischen Umweltschädigungen beziehungsweise sozialen Zwangslagen betroffen sind. Sich von den Belangen künftiger Generationen betroffen zu fühlen, ist deutlich schwieriger. Dies liegt an einer prinzipiellen Hier-und-Jetzt-Orientierung, welche sich genetisch-dispositiv erklärt. Chancenreich ist in diesem Kontext eine Anregung selbstreflexiver Prozesse.

\section{Bankberatung zwischen Anspruch und Realität}

Förderlich für eine weitere Emanzipation zukunftsverantwortlicher Investments ist eine Anlageberatung, bei der umweltethische Anliegen von Kunden explizit berücksichtigt werden. Diesem Anspruch steht jedoch eine verbreitete Beratungspraxis gegenüber, die ausschließlich ökonomische Motive in den Mittelpunkt stellt. Gemäß finanztheoretischen Ausgangsbasen sind Kapitalanleger(innen) daran interessiert, die drei Anlageziele Rentabilität, Liquidität und Sicherheit in maximaler Ausprägung zu realisieren. Das Anlageobjekt sollte im Idealfall hohe Gewinnausschüttungen und Kapitalzuwächse bei geringem Verlustrisiko und einer jederzeitigen Verfügbarkeit des angelegten Geldes mit sich bringen. Der Ausgleich dieser konkurrierenden Anlageziele erweist sich in der Beratungspraxis als äußerst schwierig. Von Bankberatern wird daher zur Er- mittlung des anlegerspezifischen Risikoprofils ein Kundenbefragungsbogen gemäß Wertpapierhandelsgesetz verwendet ( \31-33 WpHG). Der Befragungsbogen beinhaltet jedoch nicht den für ethisch-ökologische Investments bedeutsamen Aspekt der Mittelverwendung, obwohl dieser für etliche Anleger(innen) durchaus als wichtig erachtet wird.

Raubbau an natürlichen Ressourcen, Menschenrechtsverletzungen oder Waffenherstellung sind mögliche Ausschlusskriterien, welche bei der Verwendung von Finanzmitteln relevant sein können. Als Positivkriterien gelten ökologische Produktgestaltung, fairer Handel, Frauenförderung im Betrieb etc. Wie den meisten Bankkunden bekannt ist, werden sie nach ihrer Risikofreudigkeit befragt und der Berater schlägt aus dem Spektrum der Anlagemöglichkeiten von extrem sicher bis spekulativ ein entsprechendes Produkt vor. Umweltethische Risiken können für viele Kunden in Abhängigkeit von ihrer Umwelteinstellung sicherlich ein zusätzliches Entscheidungskriterium darstellen, werden aber nur selten angesprochen. Konkrete Angebote für ethisch-ökologische Finanzprodukte bleiben in aller Regel aus. Wie lässt sich das erklären? Wenn die Mittelverwendung als viertes Anlageziel hinzukommt, erhöht sich der Beratungsanspruch. Der damit einhergehende Zeit- und Schulungsaufwand steht nicht im Interesse konventioneller Banken, was angesichts des hohen Rationalisierungsdrucks in der Finanzbranche nicht verwunderlich ist. Folglich werden bevorzugt standardisierte Hausprodukte angeboten. Nicht zuletzt hält sich bei einigen Financiers wider empirischer Evidenz das Vorurteil, der Einbezug ethisch-ökologischer Kriterien führe zur Beeinträchtigung der Wertentwicklung.

\section{Charakteristische Verhaltensmuster von Anleger(innen)}

Die Beschäftigung mit umweltethisch anspruchsvollen Themen bedeutet für Kunden eine gewisse Herausforderung. Es ist kaum zu erwarten, dass sie Fragestellungen zur Mittelverwendung im Bankgespräch ohne weiteres aufwerfen. Dies ist vor dem Hintergrund einer nicht alltäglichen Situation in einem zumeist sehr formalisierten und zeitlich straff organisierten Beratungsgespräch verständlich. In einer offenen und vertrauensvollen Atmosphäre wäre die Äußerung umweltethischer Ambitionen sicherlich angenehmer.

Interessante Verhaltensweisen von Anleger(innen) offenbart eine Komplexitätsbetrachtung. Bei Bankberatungsgesprächen handelt es sich um dynamische Kommunikationsprozesse, in denen eine Vielzahl von Informationen verarbeitet werden müssen. Typische Fehler im Umgang mit Komplexität sind häufig $\mathrm{zu}$ beobachten. Privatinvestoren neigen beispielsweise dazu, Neben- und Fernwirkungen der eigenen Anlageentscheidung zu übersehen. Sie ziehen einen Kauf ethisch-ökologischer Finanzprodukte nicht in Erwägung, weil ihnen schlicht und einfach die Einsicht fehlt, dass sie darüber zur Neubestimmung unternehmerischer Zukunftsverantwortung beitragen können (siehe Abbildung 2). 
Es zeigt sich immer wieder, dass in Anlagegesprächen die Rendite als Kernvariable angesehen wird, ohne darüber hinaus die Weiterverwertung des Geldes zu hinterfragen. Eine solche Zentralreduktion ist symptomatisch für den Umgang mit komplexen Problemen. Dietrich Dörner, der sich mit den Unzulänglichkeiten im menschlichen Denken beschäftigt, bringt es auf den Punkt: Zum einen wird durch Zentralreduktionen eine ganze Menge an weiterer Analysetätigkeit und Informationsverarbeitung eingespart. Zum anderen muss man sich über weiterreichende Konsequenzen seiner Entscheidung keine Gedanken machen und bewahrt das beruhigende Gefühl, die Dinge im Griff zu haben (Dörner 2005).

Übertriebene Vereinfachungen von Zusammenhängen und Ausblendungen von Vernetzungen bei der persönlichen Kapitalanlageentscheidung sind zwar sehr bequem, nicht aber einer Durchsetzung persönlicher Ethikansprüche dienlich. Diese kämen spätestens dann deutlich zum Tragen, wenn Bankberater fragen würden: „Möchten Sie mit Ihrer Geldanlage Gentechnik, Atomkraft und Kinderarbeit unterstützen?" Eine ähnliche, allerdings weitaus weniger pointierte Frage fordert das britische Researchinstitut Ethical Investment Research Service (EIRIS) schon länger verbindlich ein: „Do you wish your investments to take into account ethical, environment or social issues and if so to what extent?" (Forum Nachhaltige Geldanlagen/ Eurosif 2002).

\section{Konsequenzen und Perspektiven}

Der Engpass für die Entwicklung des Marktes ethisch-ökologischer Finanzprodukte kann durch die stärkere Berücksichtigung verhaltensdeterminierender Faktoren überwunden werden. Aufgabe der Vorreiter in diesem Anlagesegment sollte es sein, die Transaktionskosten für den potenziellen Kunden möglichst gering zu halten. So ist eine bequemere Erreichbarkeit speziell Grüner Finanzdienstleister anzustreben und eine unkomplizierte Abwicklung entsprechender Finanzgeschäfte zu gewährleisten. In der Quintessenz ist eine umweltorientierte Anlageentscheidung umso wahrscheinlicher, je geringer die damit verbundenen Kosten sind. Dabei handelt es sich nicht nur um monetär operationalisierbare, sondern auch um oft unterschätzte gefühlte Kosten. Im Vertrieb ethisch-ökologischer Finanzprodukte sollte besonderes Augenmerk auf emotionale Faktoren gelegt werden. Das sprichwörtliche gute Gewissen ist zweifelsfrei für viele Anleger ein wichtiger Gesichtspunkt, sodass sich moralische Appelle durchaus als probates Mittel erweisen können.

Zur Abschwächung der ökologischen Verhaltenslücke bei der Kapitalanlage muss auch die Informationstransparenz erhöht werden, zum Beispiel über eine offensivere Kommunikationspolitik. Nach Möglichkeit sollten so viel Informationen an die potenziellen Investoren heranzutragen werden, dass ethisch-ökologische Finanzprodukte im günstigsten Fall bereits vor der eigentlichen Geldanlageentscheidung präferiert werden. Eine nachvollziehbare Darstellung der Lenkungswirkung, die
Abbildung 2: Lenkungswirkung ethisch-ökologischer Finanzengagements

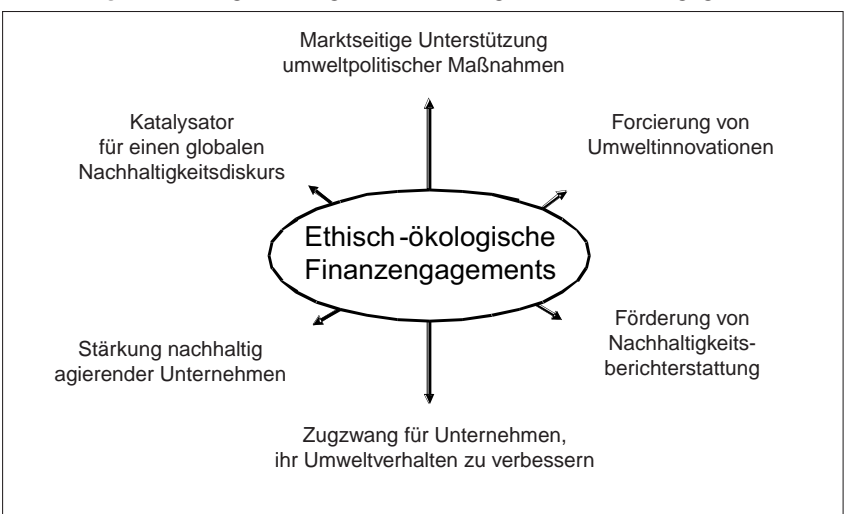

Quelle: eigene Darstellung

durch entsprechende Finanzengagements ausgelöst wird, ist in diesem Zusammenhang von herausragender Bedeutung. Ecopreneuren und Verfechtern dieser Anlageform kommt dabei eine entscheidende Multiplikatorfunktion zu.

Vermutlich ist die individualisierte Kommunikation der unmittelbaren Betroffenheit letztlich ausschlaggebend für ein Engagement. Die Qualität der persönlichen Anlageberatung hat dabei einen zentralen Stellenwert. Im Bankgespräch sollte die Tragweite der Geldanlage thematisiert werden. Nahezu alle konventionellen Banken haben sich dem Leitbild der Nachhaltigen Entwicklung verschrieben. Ist die Auseinandersetzung mit Fragen zur Mittelverwendung damit nicht selbstverständlich? Gestaltungsspielräume gibt es zu Genüge, so kann etwa der Kundenbefragungsbogen erweitert werden. Ansätze hierfür bietet Artikel 19, der im April 2004 durch die Europäische Union verabschiedeten Directive on Markets in Financial Instruments (2004/39/EC).

Die Beachtung umweltethischer Präferenzen von privaten Kapitalanleger(innen) wird darin implizit gefordert. Eine derartige Änderung bankpolitischer Rahmenbedingungen kann durch freiwillige Selbstverpflichtungen oder ordnungsrechtlich durch eine Modifikation der Paragrafen 31-33 WpHG geschehen. Die formalisierte Frage nach der Mittelverwendung wirkt sich gegenüber einer unstrukturierten Diskussion ethisch-ökologischer Kundenansprüche auf jeden Fall komplexitätsreduzierend aus. Dies wäre gleichzeitig einer kundengerechteren Beratungspolitik zuträglich. Anlegerseitige Unsicherheiten, die aus einer mangelnden Überschaubarkeit der Informationen resultieren und letztlich ein Zurückgreifen auf bekannte Produkte zur Folge haben, werden dadurch vermindert. Die Vorteile ethisch-ökologischer Finanzprodukte müssen prägnant und verständlich dargestellt werden, andernfalls führt die Macht der Gewohnheit zur Bevorzugung konventioneller Anlageformen.

Es gilt allgemein, ein optimales Komplexitätsniveau im Beratungsgespräch zu erreichen. Die Menge der Informationen soll zwar ausreichend und genau sein, Anleger(innen) müssen sie allerdings noch überschauen und verarbeiten können. Da eine Ex-ante-Bestimmung des optimalen Komplexitätsniveaus nicht generell möglich ist, sollte der Berater über ein hohes $\rightarrow$ 


\section{„Eine der zentralen Fragen bleibt, wie und in welcher Form das Thema Umwelt in die Köpfe der potenziellen Investoren gelangt."}

Empathievermögen verfügen. Informationen müssen je nach Fall und Situation kundengerecht aufbereitet und vermittelt werden. Die bankbetriebliche Aus- und Weiterbildung sollte nicht nur auf den Erwerb von Fachkompetenzen für ethisch-ökologische Anlagemöglichkeiten, sondern ebenfalls auf die Erlangung von Schlüsselqualifikationen abzielen.

Eine Bildung für nachhaltige Entwicklung führt zur ökologischen Sensibilisierung der Nachfrager. Hierdurch werden Bewusstsein und Verhalten zugunsten einer umweltorientierten Kaufentscheidung positiv beeinflusst. Umweltbildung sollte das systemische und vernetzte Denken fördern, um die Handhabung von Komplexität zu schulen. Für ethisch-ökologische Anlageentscheidungen spielt zusätzlich die Wertevermittlung im Sinne einer Übernahme gesellschaftlicher Verantwortung eine bedeutende Rolle. Eine ganzheitliche Erfahrungsfähigkeit, dazu gehört die Selbstreflexion des Umweltverhaltens, kann Wahrnehmungsbarrieren abbauen und ermöglicht einen nachhaltigen Umgang mit den natürlichen Ressourcen auf lokaler und globaler Ebene.

\section{Forschungsbedarf}

Bislang galt das Forschungsinteresse vorwiegend dem finanziellen Risiko von ethisch-ökologischen Finanzprodukten. Bei Vergleichen mit konventionellen Pendants wurden keine signifikanten Unterschiede in der Wertentwicklung festgestellt, was zweifellos der erfolgreichen Etablierung zukunftsverantwortlicher Anlagemöglichkeiten zugute kam. Die Auseinandersetzung mit den von ethisch-ökologischen Anlagevarianten ausgehenden Finanzrisiken ist weiterhin essenziell. Zur Durchleuchtung des ethisch-ökologischen Finanzmarktgeschehens sind verstärkt verhaltenswissenschaftliche Erklärungsansätze heranzuziehen.

Kapitalanleger(innen) handeln in der Praxis keineswegs nach einem strikt rationalen Kosten-Nutzen-Kalkül. Ihr Verhalten wird von zusätzlichen, zeitlich variablen Einflussgrößen bestimmt, die in der empirischen Kapitalmarktforschung bisher kaum Berücksichtigung fanden. Eine Zukunftsaufgabe ist der Einbezug von umweltsoziologischen und umweltpsychologischen Erkenntnissen. In der Konsequenz sollten die Zusammenhänge zwischen Einstellungs- und Verhaltensvariablen bezogen auf ethisch-ökologische Finanzprodukte theoretisch und empirisch weiterverfolgt werden. Parallel dazu wäre ein branchenübergreifendes Benchmarking aufschlussreich. Hieraus können sich konkrete Handlungsempfehlungen zur Reduzierung der ökologischen Verhaltenslücke bei der Kapitalanla- ge ergeben. Intrapsychischen Wahrnehmungs- und Bewertungsprozessen von Umweltproblemen sollte dabei besondere Aufmerksamkeit geschenkt werden, da sie das umweltbezogene Verhalten erheblich beeinflussen.

Einstellungs-Verhaltens-Zusammenhänge zeichnen sich durch ein hohes Maß an Komplexität aus. Einem ganzheitlichen Anspruch folgend, sollte versucht werden, die komplexen Ursache-Wirkungs-Beziehungen im Zusammenhang mit der ethisch-ökologischen Kapitalanlageentscheidung aufzudecken. Hierzu müssen einerseits die anlegerspezifischen Motive in ihrer Vielfalt erfasst und deren Vernetzung analysiert werden. Andererseits muss der Frage nachgegangen werden, welche Einflussfaktoren sich als Ansatzpunkte für Interventionsstrategien eignen. Eine verstärkte interdisziplinäre Zusammenarbeit zwischen Umweltverhaltens- und Kapitalmarktforschung könnte sich als Königsweg für ein Mainstreaming ethisch-ökologischer Finanzprodukte herausstellen.

\section{Fazit}

Eine der zentralen zu klärenden Fragen bleibt, wie und in welcher Form das Thema Umwelt in die Köpfe der potenziellen Investoren gelangt und von dort die individuellen Anlageentscheidungen beeinflusst. Dabei spielen verhaltensdeterminierende Einflussfaktoren eine außerordentliche Rolle. Investoren verhalten sich nach persönlicher Umwelteinstellung, wenn die ethisch-ökologische Anlagevariante bequem zugänglich ist und kein Zusatzaufwand entsteht. Anbieterseitige Bestrebungen sollten darauf hinauslaufen, die Transaktionskosten für den Investor so niedrig wie möglich zu halten. Eine höhere Informationstransparenz stellt einen weiteren Erfolgsfaktor dar. Es kommt darauf an, den Anleger emotional anzusprechen und Wissen über die nachhaltige Lenkungswirkung ethisch-ökologischer Kapitalanlagen zu vermitteln.

Je betroffener und emotional berührter der Kunde von umweltethischen Missständen ist, desto geringfügiger fällt die ökologische Verhaltenslücke ins Gewicht und entsprechende Finanzengagements sind wahrscheinlicher. Interessante Ansatzpunkte liefert eine Analyse charakteristischer Verhaltensmuster im Bankberatungsgespräch. Dieses stellt für private Anleger(innen) eine komplexe und nicht alltägliche Situation dar. Im Kundenverhalten sind häufig Unsicherheiten und typische Fehler im Umgang mit Komplexität ersichtlich, was zum Übersehen von Neben- und Fernwirkungen, Zentralreduktionen und Zurückgreifen auf Bekanntes führen kann. Die Kommunikation der Mittelverwendung ist schwierig. Ein kundenspezifisch optimales Komplexitätsniveau kann Abhilfe schaffen. Erforderlich ist eine fachliche und soziale Kompetenz des Anlageberaters, die gleichfalls im Zeichen einer guten Beratungspraxis steht.

Insgesamt bietet die Identifizierung und geeignete Handhabung von verhaltensbestimmenden Einflussgrößen neue Chancen auf dem Wege einer besseren Marktentwicklung ethischökologischer Finanzprodukte. Die konventionellen Banken 
sollten eine nachhaltigere Beratungspolitik anstreben, um den umweltethischen Kundenansprüchen gerecht zu werden. Ebenso wäre eine intensive Auseinandersetzung der interessierten Kreise im Rahmen eines interdiziplinären, denkschulenübergreifenden Diskurses wünschenswert. Setzt sich der Trend zu einer integrativen und damit ganzheitlichen Risikobetrachtung fort, dürfte sich eine nachhaltige und damit zeitgemäße Finanzanalyse bald als Selbstverständlichkeit erweisen.

\section{Literatur}

Avanzi SRI Research/ SiRi Group: Green, social and ethical funds in Europe. Milano 2005. Download unter www.avanzi-sri.org

Brucks, W. M.: Kollektive Ressourcenbewirtschaftung aus sozialpsychologischer Perspektive. Lengerich 2004.

Bundesministerium für Umwelt, Naturschutz und Reaktorsicherheit (Hrsg.): Umweltbewusstsein in Deutschland 2004. Berlin 2004. Download unter www.bmu.de

Deml, M./ May, H.: Grünes Geld - Jahrbuch für ethisch-ökologische Geldanlagen 2005/2006. Stuttgart 2005.

Diekmann, A./ Preisendörfer, P.: Umweltsoziologie - Eine Einführung. Reinbek 2001.

Dörner, D.: Die Logik des Misslingens - Strategisches Denken in komplexen Situationen. Reinbek 2005.

Forum Nachhaltige Geldanlagen/ Eurosif: SRI - Task or Tool for Public Policy. Summary Report of the Eurosif Conference. Frankfurt 2002. Download unter www.eurosif.org

Günther, E./ Günther, T./ Hoppe, H.: Are environmental aspects value drivers for companies? A review of empirical studies. Dresdner Beiträge zur Betriebswirtschaftslehre Nr. 81/04. Dresden 2004.

Schäfer, H./ Stederoth, R.: Portfolioselektion und Anlagepolitik mittels EthikFiltern - ein Überblick zum Stand der empirischen Kapitalmarktforschung. In: Ehrlicher, W./ Francke, H. H./ Krümmel, H. J./ Rudolph, B. (Hrsg.): Kredit und Kapital. Heft 1/02. Bonn 2002. S. 101-148.

Schoenheit, I.: Markttransparenz im Social Responsible Investment - Konsequenzen für eine nachhaltige Erwachsenenbildung. Frankfurt am Main 2005.

Stitzel, M.: Wie schlecht ist der Mensch? Eine Kritik ökonomischer Welt- und Menschenbilder. In: Burkhart, H./ Gronke, H. (Hrsg.): Philosophieren aus dem Diskurs - Beiträge zur Diskurspragmatik. Berlin 2002. S. 471-483.

\section{Anmerkungen}

(1) Eine aufschlussreiche Hinterfragung ökonomischer Menschenbilder findet sich bei Stitzel 2002.

(2) Für eine umfassende Darstellung Grüner Finanzdienstleister inklusive Kontaktdaten siehe Deml/ May 2005.

\section{AUTOREN + KONTAKT}

Diplom-Kaufmann Uwe Demele ist externer Doktorand am Lehrstuhl von Prof. Dr. Michael Stitzel (FU Berlin) und Dozent an der FHTW Berlin.

FHTW Berlin (FB 3), Treskowallee 8, 10318 Berlin. Tel. 030/42087236, E-Mail: uwedemele@web.de

Diplom-Kaufmann Gabriel Weber ist Arbeit suchender Masterstudent an der Forschungsstelle für Umweltpolitik der FU Berlin.

Wühlischstraße 56, 10245 Berlin. Tel. 030/ 78086567, E-Mail: gabrielweber@o2online.de

\section{Fachhochschule}

Münster University of Applied Sciences

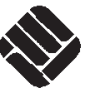

Die Fachhochschule Münster ist bundesweit eine der größten und erfolgreichsten Hochschulen für Angewandte Wissenschaften. Ihr Fachbereich Oecotrophologie ist höchst interdisziplinär aufgestellt und profiliert sich durch innovative Lehr- und Forschungsaktivitäten in den Bereichen Ernährung und Dienstleistungsmanagement. An diesem Fachbereich wird mit Unterstützung einer Gemeinschaft von Unternehmen und Stiftungen eine

\section{Stiftungsprofessur "Nachhaltige Ernährung/Ernährungsökologie"}

eingerichtet. Diese W-Professur ist zunächst auf fünf Jahre befristet zu besetzen und soll bei Etablierung des Lehr- und Forschungsgebietes verstetigt werden. Ideale Bewerberinnen und Bewerber sind im Forschungsfeld Nachhaltige Ernährung/Ernährungsökologie ausgewiesen. Es wird die Vertretung des Fachgebietes in Lehre, Wissenstransfer und Forschung erwartet. Außerdem ist die Stelle mit dem aktiven Aufbau des Masterstudiengangs "Nachhaltige Dienstleistungs- und Ernährungswirtschaft" verbunden. Im Mittelpunkt der Forschung stehen Umsetzungsprobleme der Nachhaltigen Ernährung und deren Lösung. Die Forschung soll in enger Kooperation und Zusammenarbeit mit Institutionen sowohl der Wissenschaft als auch der Wirtschaftsförderung und des Wissens- und Technologietransfers erfolgen.

Die Bereitschaft zur interdisziplinären Zusammenarbeit und zur Anknüpfung des Lehrgebietes an die Lehramtsstudiengänge des Fachbereichs im Gebiet "Ernährungsund Hauswirtschaftswissenschaft" wird vorausgesetzt.

Die Befähigung zur Lehre in englischer Sprache ist unbedingt erforderlich.

Allgemeine Hinweise Die Bereitschaft zur Mitarbeit in der Selbstverwaltung wird erwartet. Die Fachhochschule Münster möchte auch im Bereich von Lehre und Forschung den Frauenanteil erhöhen und fordert deshalb insbesondere Frauen zur Bewerbung auf. Schwerbehinderte werden bei gleicher Eignung bevorzugt berücksichtigt.

Die gesetzlichen Einstellungsvoraussetzungen können unter der unten angegebenen Anschrift angefordert oder unter www.fhmuenster.de abgefragt werden.

Bewerbungen sind Rektor der Fachhochschule Münster bis zum 03.04.2006 Hüfferstr. 27

zu richten an den D-48149 Münster 
(c) 20I0 Authors; licensee IÖW and oekom verlag. This is an article distributed under the terms of the Creative Commons Attribution Non-Commercial No Derivates License (http://creativecommons.org/licenses/by-nc-nd/3.o/), which permits unrestricted use, distribution, and reproduction in any medium, provided the original work is properly cited. 